\title{
Le médecin connecté
}

\section{Remo Osterwalder}

Dr méd., vice-président de la FMH, responsable du département Prestations et développement professionnel

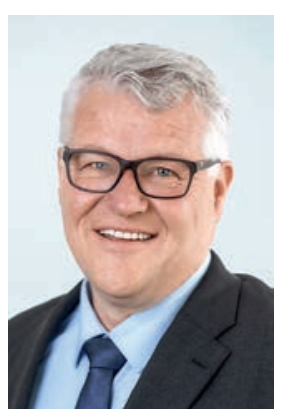

L'éditorial d'aujourd'hui passe en revue la journée de travail standard d'un médecin en mode «connecté». Le matin, le premier regard se porte sur l'iPhone. Il me réveille comme prévu, à des heures différentes selon le programme de la journée. (On peut le configurer jusqu'à une semaine à l'avance.) Au petit déjeuner, je consulte encore une fois mon agenda sur mon ordinateur portable. Celui-ci accède directement au serveur du cabinet et me permet une organisation rigoureuse des prochaines heures.

$\mathrm{Au}$ moment de quitter la maison, je reçois un texto m'informant de la circulation pour rejoindre mon cabinet. Un service que mon appareil a adopté tout seul grâce à l'application de reconnaissance géographique de mes mouvements les plus fréquents. Arrivé au cabinet, j'ouvre la porte avec mon badge, laissant là aussi une empreinte virtuelle de ma vie quotidienne. Surprenant aussi que cette autorisation d'accès comportant mon nom, l'heure et le lieu (la porte exacte) soit sauvegardée aux Pays-Bas. Qui pourrait utiliser ces données et de quelle manière, je n'en ai pas la moindre idée.

A peine sur Internet, et déjà de la publicité qui apparaît et attire mon attention sur de nouveaux appareils médicaux.

Ensuite, je me connecte à mon ordinateur portable, laissant une nouvelle trace électronique. Vite, encore un bref passage sur Internet; tiens justement, voilà une publicité pour de nouveaux appareils médicaux. Cela ne me choque même plus, depuis bien longtemps d'ailleurs. C'est simplement parce que la veille, j'avais lancé une recherche à ce sujet sur Internet. Quelle bienveillance de la part du WWW de m'inonder d'informations de ce genre (comprenez-moi bien, ceci est ironique). Avant la première consultation, je passe encore rapidement un coup de téléphone via Fixnet. Récemment, j'ai été informé que ses services ont été équipés d'une empreinte vocale. En d'autres termes, ils peuvent reconnaître mon identité par le son de ma voix - good vibrations?

Et ce n'est pas tout. Durant de nombreuses heures, j'alimente mon ordinateur avec des données sur l'état de santé de mes patients. Ces informations restent sur le serveur du cabinet et sont confidentielles. Une situation qui devrait changer dès que les premiers patients voudront basculer vers le dossier électronique. J'espère juste que mes tâches administratives n'augmenteront pas encore un peu plus. Comme ces données sortiront aussi du cabinet, je dirai à mon personnel de vérifier deux, voire trois fois l'archivage pour qu'aucun document ne se retrouve malencontreusement dans le dossier d'un autre. Cela signifie aussi que je devrai engager davantage de personnel.

Les données des patients restent sur le serveur du cabinet. Mais cela va changer dès que les premiers patients voudront passer au dossier électronique.

Travaillant à l'hôpital l'après-midi, je quitte le cabinet vers $12 \mathrm{~h}$ et reçoit aussitôt un texto amical sur l'état des routes pour mon trajet de 20 minutes. Sur place, même procédure avec le badge et la connexion au réseau de l'hôpital. Le premier rendez-vous sur mon agenda consiste en une discussion en lien avec une analyse génétique pour savoir si le patient serait prêt à participer à une étude internationale. Comme les informations le concernant devraient être transmises à une autre institution, j'ai besoin de son accord. Cela implique bien évidemment aussi que je l'informe précisément de l'utilisation de ses données: la gestion centrale des données est installée à Bruxelles et l'équipe qui mène l'étude est détachée dans cinq instituts répartis dans le monde entier.

A 18h, je quitte la clinique, naturellement dans le respect de la procédure. Je me dépêche pour passer encore par le centre commercial. Comme je n'ai pas le temps de retirer d'espèces, je paie avec PayPal sur mon téléphone, c'est quand même bien pratique. Mais voilà, un changement devrait intervenir à partir de 2018 dans l'espace UE. Cette application, et donc mon téléphone aussi, aura accès directement à mon compte bancaire. Je suis impatient de savoir comment ça va évoluer. J'espère simplement que je ne perdrai pas un jour mon emploi à cause de mon portable parce qu'il aura aussi repris ma propre fonction. 